\title{
FLIGHT SIMULATION OF MULTIPLE AIRCRAFT AND HELICOPTERS USING A SINGLE HIGH-PERFORMANCE COMPUTER
}

\section{R. M. HOWE*}

The University of Michigan, Ann Arbor, Michigan

Applied Dynamics International, Ann Arbor, Michigan

\begin{abstract}
This paper describes the simultaneous simulation of multiple airframes in real time using a single high-speed computer, the $A D$ 100. Examples include the simulation of up to 32 aircraft over their full flight envelopes, 8 helicopters with each helicopter simulated using the blade element method, the simultaneous simulation of multiple fighter aircraft together with six-degree-of-freedom missile simulations, and the simultaneous simulation of airframes and turbofan engine dynamics. A simple method for handling separate integration mode control (reset. oper ate, hold) for each of the airframes is also described.
\end{abstract}

\section{Introduction}

Flight simulation of the engagement of multiple piloted aircraft for both training and weapons system evaluation is becoming widespread. Full flight envelope simulation of high-performance aircraft requires large numbers of multivariable aerodynamic functions, which can consume considerable processor time on general purpose computers. If the flight control system is also included in the simulation, the required integration frame rates for satisfactory dynamic accuracy can become quite high. The net result is that a single, comprehensive airframe simulation can tax the speed capabilities of very fast general purpose computers. In fact, there are a number of examples where two or more general purpose computers have been required for the real-time simulation of a single aircraft. It follows that the simulation of multiple airframes can require a large number of general purposes computers, which in turn can introduce programming and timing problems, especially in a realtime environment.

In this paper we describe the use of the Applied Dynamics $A D 100$ computer for the simulation of airframes. The $A D 100$ is a multiprocessor with special architecture and software which has been optimized for the solution of ordinary, nonlinear differential equations. The computer uses emittercoupled (ECL) logic with floating-point ward lengths of 56 bits and 65 bits. It can perform 10 million multiplies and 10 million adds per second. The total time required for a 56 bit floating point multiply is 0.075 microseconds, and for a 65 bit floating point add is 0.1 microseconds. The eqivalent overall instruction rate exceeds 100 million instructions per second. Because of the short multiply and add times, and the special architecture, the $A D 100$ is extremely fast in the solution of scalar problems. The Function Memory Unit (FMU) in the $A D 100$ includes a solid-state memory of 2.097 million 65 bit words and is designed to be especially efficient in the generation of multivariable functions. The $A D 100$ interface can handle up to 5 megawords per second. In the examples which follow we will see how the above characteristics can be utilized in the real-time simulation of multiple complex airframes within only one AD 100.

\section{Multiple Aircraft Simulation}

As a first example we consider the simulation of an aircraft with six degrees of freedom, along with a simplified flight control system. The rotational equations of motion are written using aircraft body axes. while the translational equations of motion are written using flight-path axes. 1 Quaternions are used to represent the angular orientation of the aircraft. Conventional Euler angles are computed from the quaternions for display purposes. There are 13 state variables associated with the six degrees of freedom of the rigid airframe (the 4 quaternions introduce a redundant state). The flight control system includes 7 state variables and six limiter-type nonlinearities. The count of multivariable functions used in the simulation to represent aerodynamic coefficients is the following:

$$
\begin{aligned}
& 2 \text { one-variable functions } \\
& 5 \text { two-variable functions } \\
& 3 \text { three-variable functions } \\
& 6 \text { four-variable functions }
\end{aligned}
$$

The time required on the $A D 100$ for a single pass through the airframe equations (i.e., one integration step when using a single-pass integration method) is 128.7 microseconds. The highest frequency in the simulation is the 5 hertz frequency of the control-surface actuators. With the $A B-2$ (second-order AdamsBashforth) integration algorithm, an integration step size of 8 milliseconds gives a dynamic accuracy of roughly one percent in the actuator simulation. 2 The highest frequency for the rigid airframe in our example here is the 1 hertz associated with the short-period longitudinal motion at maximim dynamic pressure. In this case the dynamic error resulting from the 8 millisecond integration step size will be much less than one percent. Since the AD 100 requires only 128.7 microseconds for one integration step. the aircraft simulation can be run at $8000 / 128.7$ or 62.16 times 
real time. The use of multiple frame-rate integration for the 5 hertz actuator loops could be used to fur ther speed up the simulation.

It should be noted that the number of multivariable aerodynamic functions required for a full flight envelope simulation of a complex, high performance aircraft may be considerably larger than the function count listed above. Table 1 shows a tabulation of the exection times required by the AD 100 for the computation of multivariable functions using table lookup and linear interpolation. The table consists of two sections. The first section shows the execution times for the binary search required to identify the largest breakpoint contained in each function input variable. Three binary searches are mechanized simultaneously, with the total execution time for each trio dependant on the number of breakpoints. The second section lists the total execution time for function evaluation using linear interpolation. For example, the 16 aerodynamic functions listed above have 8 different input variables which require the following binary search kernals: one 65-breakpoint kernal (2.8 microseconds), one 17breakpoint kernal (2.2 microseconds) and two 9breakpoint kernals (3.8 microseconds) for a total execution time of 8.8 microseconds. Total execution time for the function evaluation kernals is given by $2(.6)+5(1.1)+3(1.9)+6(3.5)=33.4$ microseconds Thus the overall AD 100 execution time for computing the 16 multivariable aerodynamic functions in our example aircraft simulation is $8.8+33.4=42.2 \mathrm{mi}-$ croseconds. It is clear from this example and Table 1 that a much larger number of multivariable functions could be handled by the $A D 100$ and still have the overall integration frame time remain under several hundred microseconds.

Table 1. AD 100 execution times for multivariable function generation

\section{Binary Search Kernals}

3 binary searches for

3 binary searches for 5 breakpoints

3 binary searches for 9 breakpoints

3 binary searches for 17 breakpoints

3 binary searches for 33 breakpoints

3 binary searches for 65 breakpoints

3 binary searches for 129 breakpoints

3 binary searches for 257 breakpoints

1.3 microsec.

1.6 microsec.

1.9 microsec.

2.2 microsec.

2.5 microsec.

2.8 microsec.

3.1 microsec.

3.4 microsec.

\section{Function Evaluation Kernals}

Evaluation of a 1 -variable function Evaluation of a 2 -variable function Evaluation of a 3-variable function Evaluation of a 4-variable function Evaluation of a 5-variable function Evaluation of a 5 -variable function Evaluation of a 7-variable function

6 microsec. 1.1 microsec.

1.9 microsec.

3.5 microsec.

6.9 microsec.

13.5 microsec.

26.5 microsec.

It should be noted that the 128.7 microsecond frame time for the aircraft example described above includes 17.5 microseconds of overhead associated with the AD 100 simulation executive, called SIMEXEC. Thus the net execution time per integration frame is $128.7-17.5$ or 111.2 microseconds. This means that each additional airframe in a multiple aircraft simu- lation will require only 111.2 microseconds. For illustrative purposes, let us assume that a more complex airframe simulation requires three times the 16 multi-variable functions listed at the beginning of this section (this adds 66.8 microseconds). Let us also assume that a much more complex flight control system adds another 50 microseconds, and that 100 input/output channels add still another 20 microseconds. Then the overall integration frame time for each aircraft would be $111.2+66.8+50+20=248$ microseconds. If the required real-time step size is 8 milliseconds, as assumed above, then the $A D 100$ could in principle simulate up to $8000 / 248$ or 32 aircraft simultaneously in real time. As noted earlier, the use of multiple integration frame rates for the highfrequency flight control subsystems would permit an overall real-time step size considerably larger than 8 milliseconds. This would in turn increase even further the number of simultaneous aircraft which could be simulated.

\section{Separate Mode Control for Individual Airframes}

In a multiple engagement simulation it may be desirable to control separately in real time the integrator modes (reset, operate, hold) for each individual airframe. In the AO 100 this can be accomplished by appropriate modification of SIMEXEC. Another approach is to maintain the simulation of every aircraft in the operate mode at all times, but with the integration formulas for each state variable modified to accomplish individual mode control. To illustrate the method, assume that we are using the second-order predictor algorithm, $A B-2$, which is one of the most popular real-time methods. Then the difference equation for integrating the state equation, $d X / d t=F$, is given by

$$
x_{n+1}=x_{n}+.5 h *\left(3 * F_{n}-F_{n-1}\right)
$$

where $h$ is the integration step size. To allow separate mode control we modify the difference equation to the following form:

$$
X_{n+1}=X_{n}+K 1 * .5 h *\left(3 * F_{n}-F_{n-1}\right)+K 2 *\left(X_{0}-X_{n}\right)
$$

The constants $K 1$ and $K 2$, in the form of real-time inputs each integration frame, control the integrator mode. Thus with $K 1=1$ and $K 2=0$ the integrator is in the operate mode. With $K 1=0$ and $K 2=0$, the integrator is in the hold mode. Finally, with $\mathrm{K} 1=0$ and $K 2=1$, the integrator is in the reset mode, where in the next step the state $X_{n+1}$ will take on the initial condition $X_{0}$. If each integration for a given airframe simulation is programmed with this formula, the integration modes for that airframe can be controlled separately from all other airframes by appropriate setting of the real-time inputs $K 1$ and $K 2$.

Note that in either the reset or hold mode, the state variable derivative $F$ will be evaluated each frame using the same fixed state variables. Thus $F_{n-1}$ will be equal to $F_{n}$. When the integration is then switched to the operate mode with $K 1=1$ and $K 2=0$, the difference equation for the first integration step will be given by 


$$
x_{n+1}=x_{n}+.5 h *\left(3 * F_{n}-F_{n}\right)=x_{n}+h * F_{n}
$$

which is simply Euler integration. Subsequent steps, for which $F_{n^{+1}}$ in general is not equal to $F_{n}$. will revert to $A B-2$ integration. Actually, the usual startup method for $A B-2$ integration is Euler for the first step, so that the above technique should not cause any significant error in a real-time environment.

\section{Multiple Helicopter Simulation}

Our next example is the real-time simulation a helicopter using the blade element method. In the blade element method each rotor blade is divided into a number of segments or elements. The aerodynamic lift, drag and moment acting on each segment are calculated from the angle of attack and Mach number of the segments using two-variable aerodynamic functions. These forces and moments are then summed to obtain the overall aerodynamic forces and moments acting on the blade, which are used to integrate the blade equations of motion and to calculate the rotor forces acting on the airframe.

The angle of attack and Mach number at each blade segment are determined by computing the velocity components of the segment center of pressure with respect to the local air. These velocity components depend not only on the flapping and lagging motion of the blade, but also the rotor inflow and the translational and rotational velocities and accelerations of the airframe. The overall calculations are very computationally intensive. ${ }^{3}$ The real-time simulation is fur ther complicated by the need to use high integration frame rates. For reasonable performance and dynamic handling accuracy Houck has shown that 4 to 6 segments per blade are required, and that each integration step should correspond to no more than 12 to 18 degrees of azimuthal motion of the rotor. ${ }^{4}$ For a rotor with an angular frequency of 27 radians per second this translates into an integration step size of between 8 and 12 milliseconds for the rotor simulation.

The equations of motion of the Sikorsky UH-6DA helicopter have been programmed on the AD $100 \mathrm{com}-$ puter using the Sikorsky Gen Hel engineering simulation program. ${ }^{5}$ The simulation includes the main rotor, tail rotor, empennage, fuselage, flight controls, landing gear, engine/fuel control, and ground effects. Overall frame time on the $A D 100$ is approximately 1 millisecond. It follows that up to 8 such helicopter simulations could be run in real time on a single $A D$ 100.

It should be noted here that the $A D 100$ uses a programming language called ADSIM. which is similar to such continuous system simulation languages as ACSL and CSSL. In the current version 5.0 of ADSIM a real-time multiple airframe simulation is limited more by the size of the ECL progr am memory in the AD 100 than the speed requirements. Under version 5.0 of ADSIM and with the current size of program memory in the $A D$ 100, the UH-6DA simulation described above would be limited to 4 airframes. Version 6.0 of ADSIM. which is about to be released, will support hardware subroutining as well as the software subroutining currently supported by version 5.0. This will then remove any size restrictions in the real-time simulation of multiple airframes.

\section{Simulation of Multiple Airframes and Missiles}

In this section we consider a comprehensive six-degree-of-freedom simulation of a tactical missile, with the possibility of simulating multiple missiles and aircraft in multiple engagements. The example missile considered here is the Hellfire. which can be launched from conventional aircraft or helicopters against surface or air targets. The system being simulated consists of the missile airframe. guidance system and flight control system, including pneumatic servos which control each of four fins. $A$ total of 53 state variables is required in the simulation. The count of multivariable functions used in the simulation is the following:

$$
\begin{array}{cc}
18 & 1 \text {-variable functions } \\
17 & 2 \text {-variable functions } \\
7 & 3 \text {-variable functions }
\end{array}
$$

Net execution time on the $\mathrm{AD} 100$ for one integration frame is 267 microseconds. The frame time required for an accurate solution in real time is four times this. Thus up to 4 Hellfire missiles can be simulated in real time on a single $A D$ 100. More importantly, one $A D$ 100 can be used to simulate two or three missiles as well as multiple airframes. In section 2 we saw that an integration frame time of 8 milliseconds was representative of the real-time requirement for a high performance aircraft, including its flight control and avionics system. The 1 millisecond real-time frame requirement for the missile suggests that we compute 8 integration steps for the missile per integration step for the aircraft. If we simulate 2 missiles, the required $A D 100$ execution time per 8 millisecond frame of the aircraft simulation is $2(277)(8)=4432$ microseconds. This leaves $8000-4432=3568$ microseconds for simulating multiple aircraft. At 248 microseconds per aircraft, which is the AD 100 frame time we calculated at the end of Section 2, this would allow a simulation of up to $3568 / 248$ or 14 aircraft in addition to the two missiles.

Consider next the simultaneous simulation of helicopters and two missiles. In Section 4 we saw that the $A D 100$ frame time per helicopter when using the blade element method is 1 millsecond, whereas the requirement for real time is again 8 milliseconds. Now the 3568 microseconds available per 8 millisecond frame can be used to simulate three helicopters in addition to the two missiles, all with a single $A D 100$.

In the above two illustrations it should be noted that the tactical missiles have a flight time of only a few seconds. When the simulated flight of a given missile has terminated, the simulation of another missile can be initiated. Thus the capability of simulating multiple aircraft as well as two missiles simultaneously in no way precludes the overall succesive simulation of many more missiles in a prolonged engagement. 


\section{Simulation of Airframes plus Jet Engines}

Real-time high fidelity dynamic simulations of jet engines are used extensively for the design and testing of engine contrallers. In high performance aircraft the engine dynamics can inter act significantly with the airframe dynamics. This is especially true in helicopters. where engine interaction with the rotor often forces redesign of the engine control system. It is therefore desirable to be able to combine comprehensive simulations of airframe and engines in real time.

The $A D 100$ has been used extensively to simulate turbofan engines using sophisticated high-fidelity models which include compressor maps, turbine maps, etc.. Typical execution time for one integration frame for a high-bypasss turbofan is 110 microseconds. The required integration step size for a real-time simulation of the same engine is 1 millisecond. If we again use 8 milliseconds as the step size for the airframe. this suggests that we should take 8 integration steps in the engine simulation for each step in the airframe simulation. If we assume two engines per airframe. three airframes would require $2(3)(110)=660 \mathrm{mi}-$ crosecands per integration step in simulating the engines, or $8(660)=5280$ microseconds per $8 \mathrm{milli}-$ second frame for the airframes. This leaves 2720 milliseconds for simultaneous simulation of three airframes, which at 250 microseconds per airframe presents no problem for the AD 100.

\section{Conclusions}

We have seen how computers like the $A D 100$ with architecture optimized for the solution of scalar-type ordinary differential equations are capable of simulating many airframes simultaneously in real time. In particular we have shown that 30 or more six-degreeof-freedom comprehensive simulations of conventional aircraft, including flight control and avionics systems, and be simulated simultaneously in real time.

We have also shown that up to 8 helicopters can be simulated in real time using the blade element method. Engineering level six-degre-of-freedom missiles can also be simulated in real time simultaneously with the airframe simulations. Finally, comprehensive jet engine dynamic simulations can be combined with up to three real-time airframe simulations, all on a single AD 100 computer.

\section{References}

1. Fogarty, L.E., and R.M. Howe, "Computer Mechanization of Six-Degree-of-Freedom Flight Equations," Simulation Vol. 11. No. 4. Oct., 1968.

2. Howe, R.M., "Transfer Function and Characteristic Root Errors for Fixed-Step Integration Algorithms," Transactions of the Society for Computer Simulation, Vol. 2, No. 4, Dec.. 1968.

3. Howe, R.M., and L.E. Fogarty. "Computer Considerations for Real Time Simulation of a Generalized Rotor Model." NASA CR-2877. Sept., 1977.
4. Houck, J.A., "Computational Aspects of Real Time Simulation of a Rotary-Wing Aircraft," Masters Thesis, George Washington University, May. 1976.

5. Howlett, J.J.. "UH-60A Black Hawk Engineering Simulation Program," NASA CR-166309, Dec., 1981. 